\title{
Cardiac MRI assessment of myocardial perfusion
}

\author{
Yasmin S Hamirani' \& Christopher M Kramer ${ }^{* 1,2}$
}

\begin{abstract}
Coronary artery disease is the most common cause of mortality and morbidity around the globe. Assessment of myocardial perfusion to diagnose ischemia is commonly performed in symptomatic patients prior to referral for cardiac catheterization. Among other noninvasive imaging modalities, cardiac MRI (CMR) is emerging as a highly sensitive and specific test for myocardial ischemia and infarction. Resting perfusion on CMR is used to evaluate for microvascular obstruction, which is shown to predict adverse left ventricular remodeling and cardiac events after acute myocardial infarction. This article summarizes the current understanding of CMR perfusion.
\end{abstract}

\section{Background}

Despite significant improvement in the diagnostic and treatment strategies for coronary artery disease (CAD), it remains the leading cause of morbidity and mortality around the world across both genders. Significant stenosis of the coronary artery can lead to myocardial ischemia, which can be addressed medically or via invasive coronary intervention or surgery. Management is dependent upon the patient's symptoms and extent of myocardial ischemia, with the goal of alleviating symptoms and preventing myocardial infarction (MI), left ventricular (LV) dysfunction, malignant arrhythmias and cardiac death.

Noninvasive assessment is often used in symptomatic patients, prior to referral for an invasive coronary angiogram (with or without fractional flow reserve [FFR] measurement) to assess for CAD. Single-photon emission computed tomography (SPECT), contrast perfusion echocardiography, positron emission computed tomography (PET) and cardiac computed tomography perfusion imaging are all methods of assessing myocardial perfusion. However, each method has its limitations: for example, SPECT, PET and cardiac computed tomography involve radiation exposure, while contrast echocardiography is limited by difficult acoustic windows in certain patients and limited availability of the technique.

Cardiac MRI (CMR) is a rapidly growing, noninvasive, imaging modality for assessing for the presence of CAD. CMR is now considered the gold standard for cardiac function assessment. Currently available CMR scanners have the potential to acquire cardiac images with significantly improved signal to noise ratio, and spatial and temporal resolution. CMR is increasingly used to evaluate rest and stress perfusion, and to identify myocardial ischemia and infarct with a high degree of accuracy. Similarly, utility of CMR is studied in the setting of acute MI to assess for microvascular obstruction (MVO), which is shown to be associated with adverse cardiovascular events despite timely intervention.

\section{Myocardial perfusion imaging via CMR: technique}

CMR myocardial perfusion imaging is performed via first pass perfusion of the myocardium using chelated-gadolinium contrast, which is an extravascular and extracellular agent. Due to its paramagnetic effect, gadolinium alters the local magnetic field and enhances the relaxation rate of nearby

\section{KEYWORDS}

- 3D perfusion - cardiac MRI

- fractional flow reserve

- microvascular obstruction

- myocardial perfusion

- quantitative perfusion

- rest • stress 
water protons. Tissues that are well perfused show enhanced signal on T1-weighted images. With low dose gadolinium administered for perfusion imaging, the T2 and T2* effects do not dominate and, thus, do not interfere with the bright signal in the perfused tissues [1,2]. Intravascular gadolinium-based contrast agents were studied more than a decade ago and while some of them are on the market (Vasovist) in Europe, others never made it to market [3]. First pass perfusion imaging is performed as part of a vasodilator stress test (with stress and rest images) to identify ischemia and infarct (Figure 1). The strength of CMR is its ability to provide comprehensive information in regards to myocardial chamber volumes, overall LV ejection fraction and wall motion, myocardial perfusion and late gadolinium enhancement (LGE) to assess for evidence of fibrosis.

Detection of severity of epicardial CAD is the most common reason for performing CMR perfusion studies. They can also be performed at rest to assess the microvascular circulation in the setting of acute MI.

\section{- Rest perfusion}

Typically, ECG-gated T1-weighted images are obtained using gradient echo, hybrid gradient echo-planar or steady state free precession imaging. Three LV short axis slices (base, mid and apex) are obtained corresponding to the American Heart Association 16-segment model [4]. Images are acquired during the first pass of gadolinium. Rest perfusion is considered abnormal if upon gadolinium administration, a low signal on T1-weighted images is detected in a myocardial segment, which persists for a period of at least five heartbeats [5].

\section{- Stress perfusion}

The most commonly used vasodilator stress agent in clinical practice is adenosine $(140 \mu \mathrm{g} / \mathrm{kg} / \mathrm{min}$ intravenously [IV] for $3 \mathrm{~min}$ ), however, dipyridamole $(0.56-0.84 \mathrm{mg} / \mathrm{kg}$ body weight IV over 4-6 min) and regadenoson (0.4 mg IV bolus) have been used in clinical studies. The concept behind vasodilator stress imaging is that hyperemic flow is decreased in myocardial segments that are supplied by a significantly stenosed epicardial coronary artery (>50\%) (Figure 2) [6]. Some studies have used perfusion imaging during dobutamine stress CMR. In one such study performed on 455 patients scheduled for invasive coronary angiography (ICA), the addition of perfusion imaging to wall motion analysis with dobutamine improved the sensitivity from 85 to $91 \%$ at the cost of decreased specificity from 82 to $70 \%$ without changing the overall diagnostic accuracy [7]. Image quality is often degraded at the high heart rates with higher doses of dobutamine and, thus, this approach has not been widely adopted.

Resting myocardial perfusion imaging in acute $\mathrm{MI}$ to assess the microvasculature MVO on CMR can be identified by two techniques: resting perfusion imaging and by LGE images. On resting first pass perfusion imaging, MVO is characterized as areas of reduced signal intensity (hypoenhancement) [8] that persist for $>2 \mathrm{~min}$, in comparison to homogenously increased signal intensity in the normal myocardium after contrast administration. This is labeled as 'early MVO' in comparison to 'late MVO', which is seen in LGE images as an area of central hypoenhancement within the hyper enhanced region (Figure 3) [9]. The severity of hypoenhancement on perfusion images is seen to be consistent with severe microvascular damage. Both early and late MVO is shown in studies to be associated with incomplete ST segment resolution [10]. 'Early MVO' has a limitation in regards to less coverage of the LV myocardium, low signal to noise ratio and lower spatial resolution. Despite the limitations, presence of 'early MVO' in acute $\mathrm{MI}$, regardless of timely intervention with angioplasty, has been associated with lack of recovery of myocardial function. Late MVO carries with it a worse prognosis as it depicts a region of such severe microvascular damage that contrast has not yet arrived there as long as 10-20 min after infusion.

More than a decade ago, Wu et al. performed CMR in 44 patients $10 \pm 6$ days postacute MI and performed follow-up for $16 \pm 5$ months [11]. This study showed that even after controlling for infarct size, MVO remained a prognostic marker for cardiovascular events $\left(\chi^{2}=5.17\right.$; $\left.<<0.05\right)$ and was significantly associated with fibrous scar formation and LV remodeling. Similarly, Ørn et al. performed CMR on day 2 and 7,2 months and at 1 year in 42 patients post single vessel angioplasty for acute MI [9]. After adjusting for infarct size at day 2, detectable MVO at 1 week was noted to be an independent predictor of infarct size at 1 year along with increased LV volumes and lower LV ejection fraction $(\mathrm{p}=0.003)$. Another study showed that presence of MVO and LGE at 1 -week postacute MI was an indicator of no 


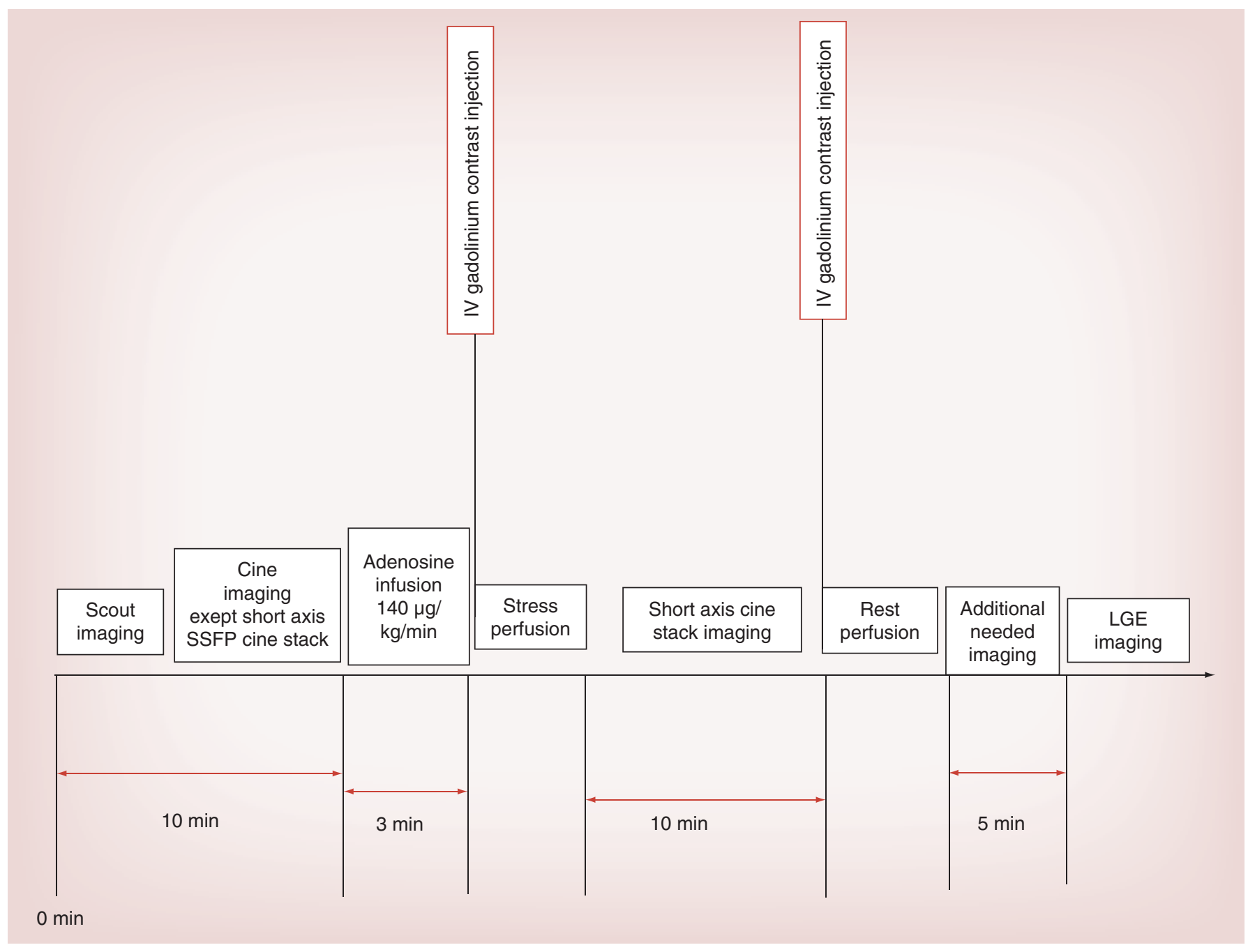

Figure 1. Vasodilator stress cardiac MRI protocol at our center.

IV: Intravenous; LGE: Late gadolinium enhancement; SSFP: Steady state free precession.

improvement in follow-up LV ejection fraction versus patients with only LGE where a partial improvement in LV function was noted at 8 weeks [12]. Lund et al. on CMR rest perfusion images, acquired $6 \pm 3$ days post MI (60 patients) revealed $\mathrm{MVO}$ to be an indicator of larger infarct size (>20\% of the LV area), reduced thrombolysis in myocardial infarction grade flow of the infarct related artery before revascularization and significantly increased peak releases of creatinine kinase and creatinine kinase-MB ( $<$ 0.001) [13].

\section{Rest-stress myocardial perfusion using \\ CMR to assess for CAD}

Vasodilator stress CMR is used to assess for CAD in symptomatic patients. After publication of multiple prospective, single center trials, Nandalur et al. published a meta-analysis in
2007 [14]. They examined stress CMR perfusion studies where ICA was used as the gold standard to detect CAD. This study showed that both a negative CMR in the presence of symptoms, and a positive CMR in intermediate and low risk patients, have high prognostic value. Recently de Jong et al. performed a comparative meta-analysis of perfusion contrast echocardiography, SPECT, PET and CMR with invasive CA as the gold standard [15]. To diagnose $>70 \%$ stenosis on coronary angiography, the comparative sensitivity and specificity of CMR, perfusion echocardiography and SPECT was: sensitivity (91\% vs $87 \%$ vs $83 \%$ ) and specificity ( $80 \%$ vs $72 \%$ vs $77 \%$ ).

Data is now available from three randomized controlled trials. (Table 1) [14,16-18]. These studies have confirmed the noninferior (and superior in some studies) performance of stress 


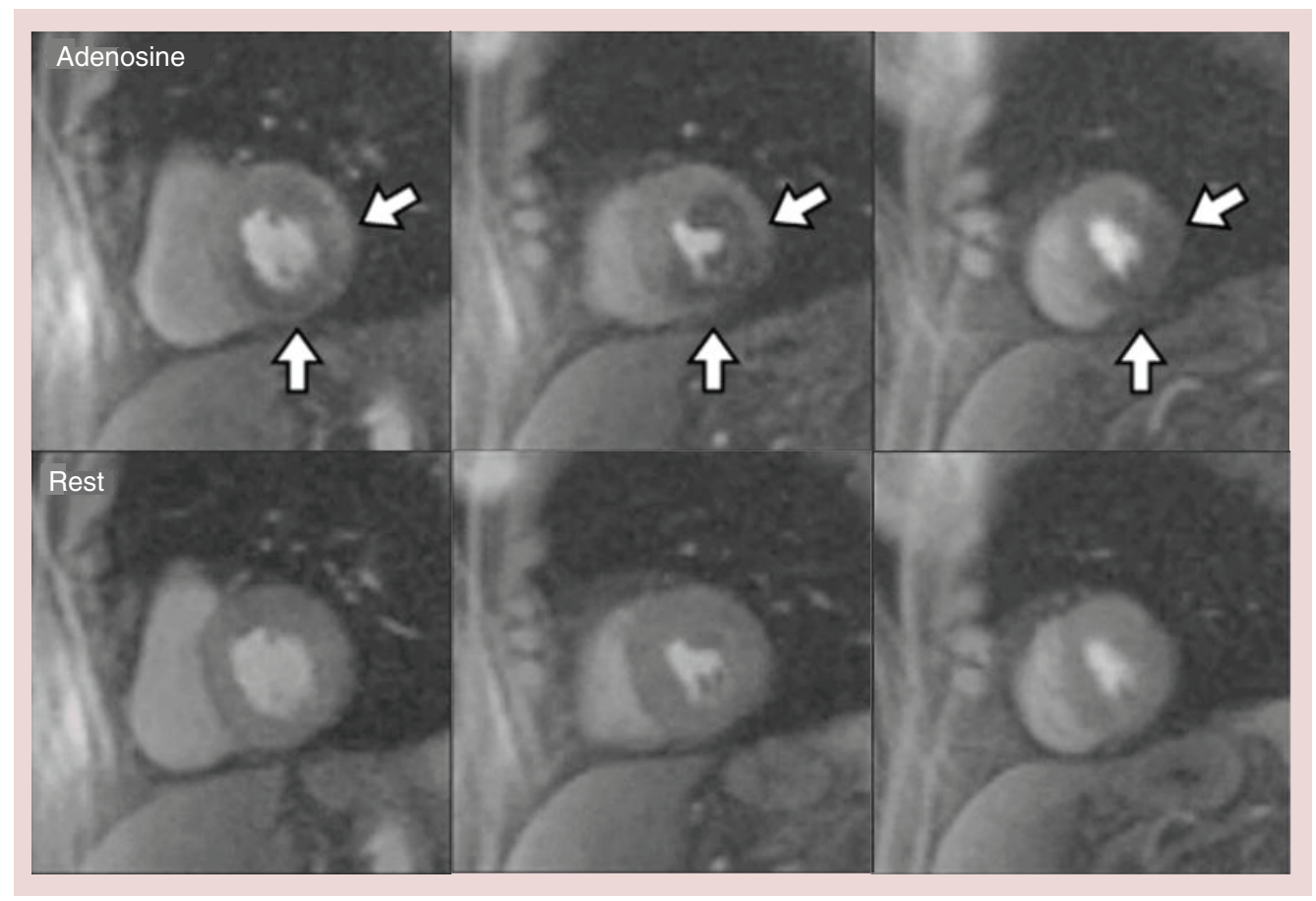

Figure 2. Qualitative interpretation of cardiac MRI perfusion imaging. Top row shows adenosine stress images, while the bottom row shows rest images. Left column: basal left ventricle; middle column: mid left ventricle; right column: apical left ventricle. The white arrows point to a large perfusion defect in the anterolateral, infero-lateral, inferior and infero-septal wall, extending from the basal to the apical slice. Adapted with permission from [6].

CMR compared to SPECT in a realistic clinical environment.

The 'MR-INFORM study' [19], is another prospective randomized controlled trial that is under progress. It aims at comparing medical treatment with coronary intervention in patients with stable CAD. Adenosine stress CMR-based coronary intervention will be compared with FFR-based coronary intervention and major adverse cardiac events over 1 year will be analyzed as the primary end point. Bettencourt et al. compared noninvasive CMR perfusion with invasive assessment of physiologic significance of a coronary lesion via FFR [20]. Their study population included symptomatic intermediate-to-high risk patients. They compared CMR perfusion with ICA and FFR with FFR cut-off of $<0.80$. In this study, in a per patient analysis the sensitivity, specificity, PPV, NPV and accuracy of stress CMR was 89, 88, 85, 91 and $88 \%$, respectively, while on a per vessel analysis they were 80, 93, 79, 94 and $90 \%$, respectively.

\section{T \& whole-heart 3D CMR perfusion}

Adenosine stress CMR perfusion imaging at 1.5 $\mathrm{T}$ is well established. However, the potential superiority of imaging at $3 \mathrm{~T}$ has recently been tested in a handful of studies and it is speculated that $3 \mathrm{~T}$ might become the preferred CMR field strength for adenosine stress CMR in clinical practice. However, more data is needed in this regard.

Manka et al. [21], found the sensitivity, specificity and accuracy of $3 \mathrm{~T}$ CMR perfusion to be 92, 74 and $83 \%$ as compared to ICA. Walcher et al. performed adenosine stress CMR on both 1.5 $\mathrm{T}$ and $3 \mathrm{~T}$ scanners, and found that the sensitivity at 3 versus $1.5 \mathrm{~T}$ was 84 versus $75 \%$ with a specificity of 90 versus $75 \%$ for $\geq 50 \%$ stenosis on ICA [22]. Similarly, when using $>70 \%$ stenosis as the reference standard, $3 \mathrm{~T}$ perfusion was found to be more sensitive (96 vs 89\%) and more specific ( 88 vs $80 \%) .3$ T whole-heart 3D CMR perfusion is another recent technical advance [23], but this was not found to be superior to traditional three-slice CMR perfusion.

\section{Quantitative perfusion analysis on CMR}

Quantification of myocardial perfusion has been an area of active research in CMR over the past few years. Myocardial blood flow increases during stress. Myocardial perfusion reserve (MPR) can 
be calculated as the ratio of stress versus resting flow. Quantitative CMR is performed to calculate absolute myocardial blood flow $(\mathrm{ml} / \mathrm{min} / \mathrm{gm})$ on dynamic first pass perfusion imaging, by various techniques including: two-compartment modeling, Fermi function modeling, modelindependent analysis and Patlak plot analysis [24]. In the Fermi model, time intensity curves are constructed in the myocardium and the arterial input function is measured from the LV cavity or ascending aorta using constrained deconvolution of myocardial signal intensity curves. The constrained deconvolution analysis uses the delay time between LV blood pool and myocardial enhancement and controls for the time duration of the plateau portion of the curve. As a final step mathematical deconvolution is performed [25]. Pack et al. compared the four models and found that global myocardial perfusion estimates at rest were similar with the four models [24]. At stress the Fermi function models was noted to give 25\% higher estimate of blood flow. Overall, MPR values with the models were not significantly different, indicating that any of them can be used for MRP measurements. A good correlation was found between PET-MPR and CMR-MPR in a study on 41 patients with known or suspected CAD ( $r=0.75 ; \mathrm{p}<0.0001)$ [26].

CMR quantitative perfusion data is now available in various populations (syndrome $\mathrm{X}$ )
[27], various myocardial regions (subepicardium vs subendocardium) [28], using different techniques (manual vs automatic) [29], and with different vasodilator agents (adenosine and regadenoson [30]).

A comparison of qualitative CMR perfusion (Q-CMR-P) and quantitative CMR perfusion analysis was performed by Patel et al. on 30 patients [6]. The sensitivity, specificity and accuracy for the assessment of $>50 \%$ stenosis on ICA of Q-CMR-P versus MPR was 79, 83 and $80 \%$ versus 88,67 and $83 \%$, respectively. A stepwise reduction in MPR was noted with increasing coronary artery stenosis, that is, MPR was $2.42 \pm 0.94$ with $<50 \%$ coronary stenosis versus $2.14 \pm 0.87$ for $50-70 \%$ stenosis versus $1.85 \pm 0.77$ for $>70 \%$ coronary stenosis. For the evaluation of myocardial ischemic burden, no difference in the Q-CMR-P was noted between triple vessel and single vessel CAD $(31 \pm 20 \%$ vs $21 \pm 26 \%, p=0.26$ ), whereas when MPR analysis was used, patients with triple vessel CAD had significantly increased ischemic burden compared with single vessel CAD (60 $\pm 38 \%$ vs $25 \pm 41 \%$ ). Thus, in this study quantitative MPR analysis was found to differentiate patients with multivessel from those with single vessel CAD (Figure 4).

The inter-study reproducibility of quantitative CMR perfusion at $3 \mathrm{~T}$ was studied by

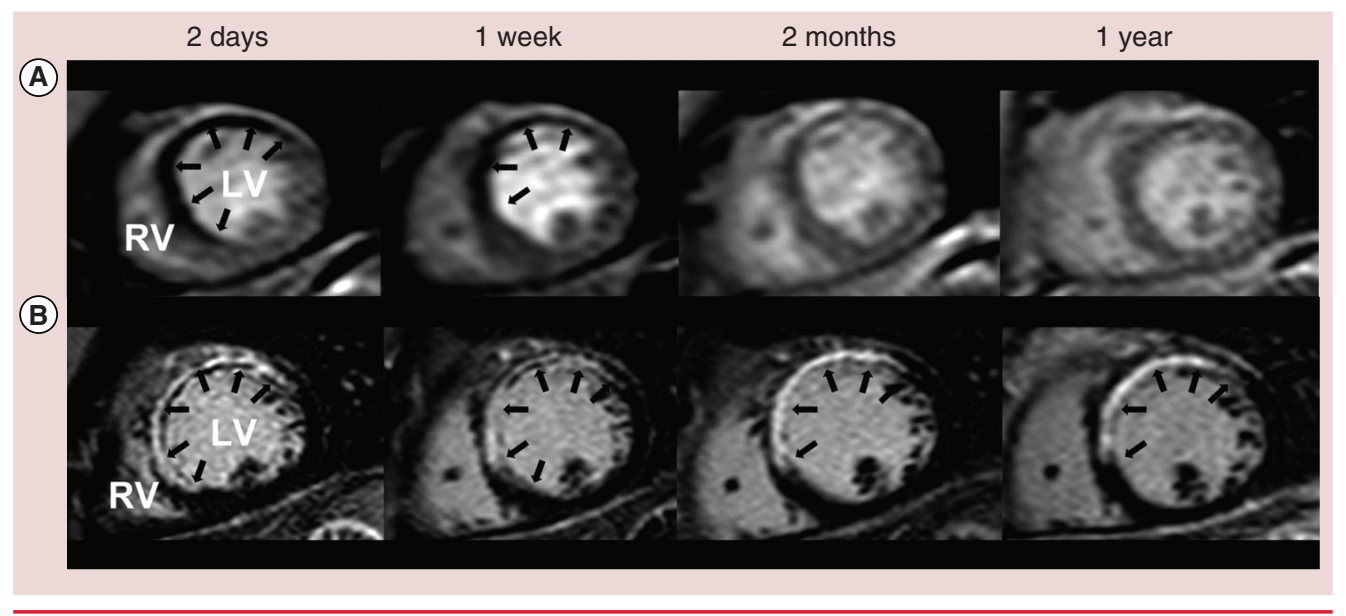

Figure 3. Typical pattern of microvascular obstruction in a patient with ST-elevation myocardial infarction due to an occluded left anterior descending artery, treated with primary percutanous coronary intervention. (A) Demonstrates first pass perfusion images with hypoperfusion of the anteroseptum, especially at day 2 and week 1 postmyocardial infarction. (B) Demonstrates late gadolinium-enhanced images with the dark signal at the subendocardium at each time point representing regions of microvascular obstruction.

LV: Left ventricle; RV: Right ventricle.

Adapted with permission from [9]. 


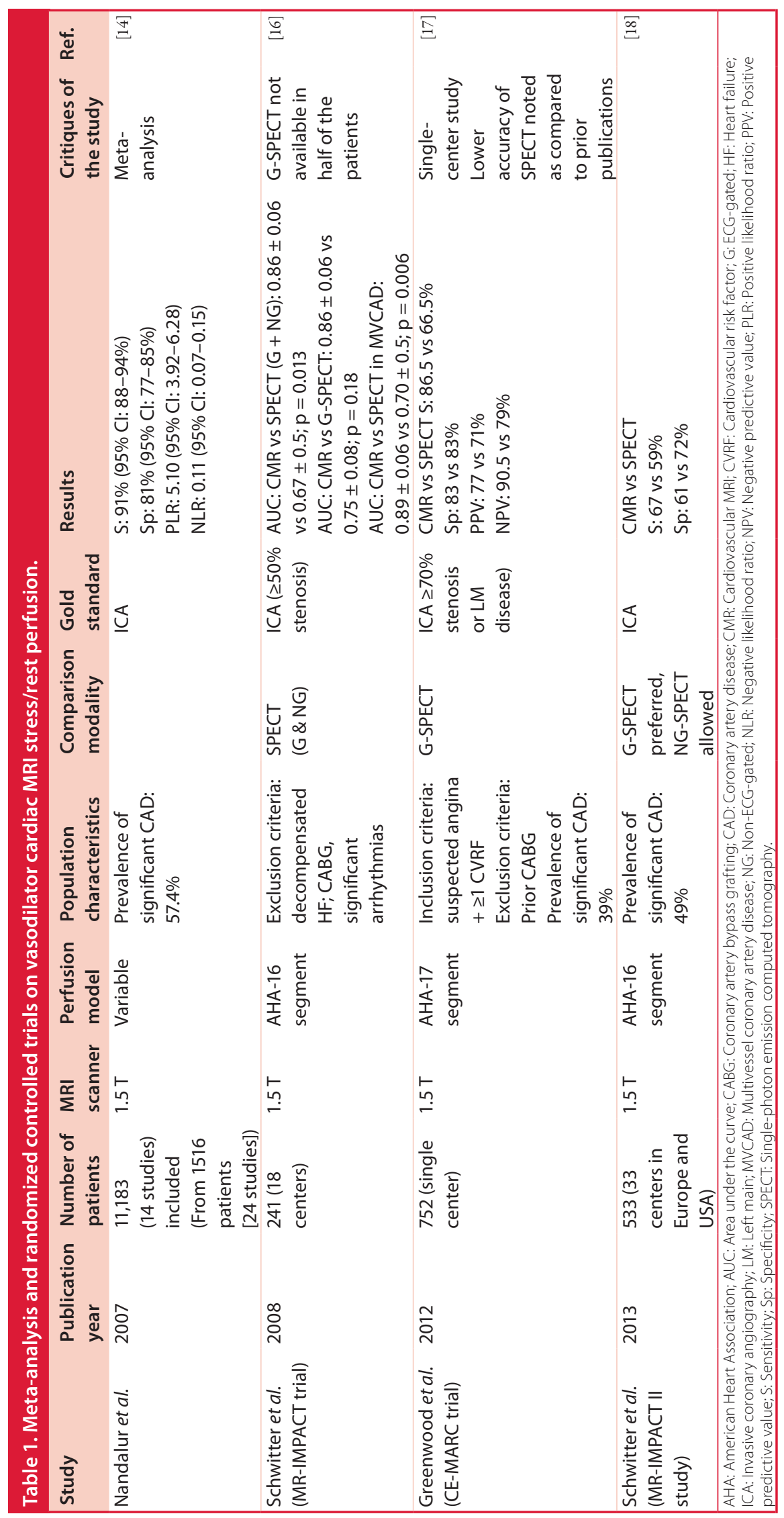


Morton et al. [31]. CMR perfusion was performed three times during a single day on 16 healthy volunteers. The global rest and stress perfusion along with MPR were comparable with a coefficient of variation $(\mathrm{CV})$ of $16.0,26.8$ and $23.9 \%$, respectively. The corresponding territorial CVs were $27.5,35.2$ and $33.5 \%$, respectively. The authors concluded that the inter-study reproducibility of quantitative myocardial perfusion is reasonable and best for global rest perfusion. However, the results appear to be dependent on the voxel size, acquisition and postprocessing methods. Misregistration and partial volume artifacts also impact the results. Fast and nearly automatic techniques are in development to quantify myocardial perfusion at both rest and stress.

\section{Latest techniques in acquiring perfusion} images on CMR

Perfusion CMR interpretation is sometimes limited by the presence of dark-rim artifacts that can lead to false-positive results. These artifacts are related to limited spatial resolution and cardiac motion. Additionally, ECG gating difficulties, especially in patients with atrial fibrillation or multiple extra atrial or ventricular beats can result in image degradation and nondiagnostic scans.

Recent technical innovations in imaging are aimed at addressing these artifacts. These alter coil encoding and spatiotemporal correlations and include: ungated, radial dynamic perfusion
[32], free breathing myocardial perfusion [33], high resolution CMR perfusion [34,35] including $\mathrm{k}-\mathrm{t}$ BLAST (Broad-use Linear Acquisition Speed-up Technique) [36] and k-t SENSE (k-space and time sensitivity encoding) [37] and sliding window conjugate gradient highly constrained back projection reconstruction (SW-CG-HYPR) [35]. Some of these techniques have resulted in significantly improved image quality with improved contrast to noise and signal to noise ratio, and have reduced the extent and transmurality of dark rim artifacts. However more work is underway to compare these to more previously used techniques.

\section{CMR myocardial perfusion \& prognosis}

In low-to-intermediate risk patients suspected of CAD, stress CMR provides prognostic information in regards to major adverse cardiac events. Nandalur's meta-analysis demonstrated that a negative stress CMR in a low to intermediate risk patient $(<60 \%$ probability of CAD) decreases the probability of CAD to $\leq 20 \%$ [14]. Similarly, in an intermediate to high risk patient $(>40 \%$ probability of CAD), when the stress CMR is positive, it increases the probability of CAD to approximately $80 \%$. The prognostic information of stress CMR was compiled in a meta-analysis from our center by Lipinski et al. [38]. Data was analyzed from 19 studies inclusive of 11,636 patients with known or suspected CAD. The

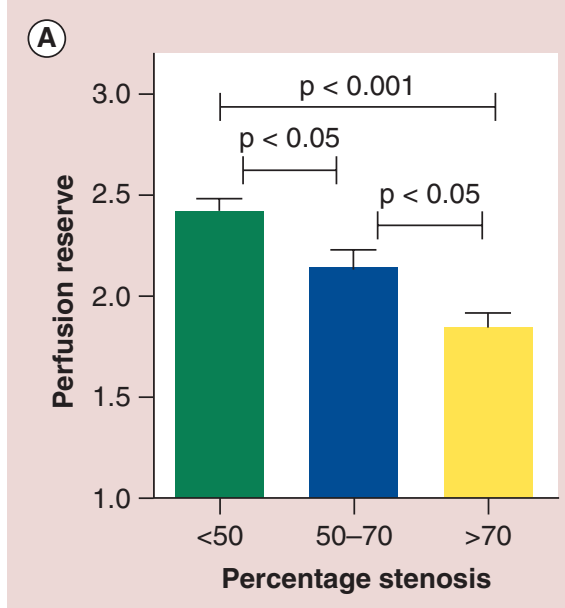

(B)

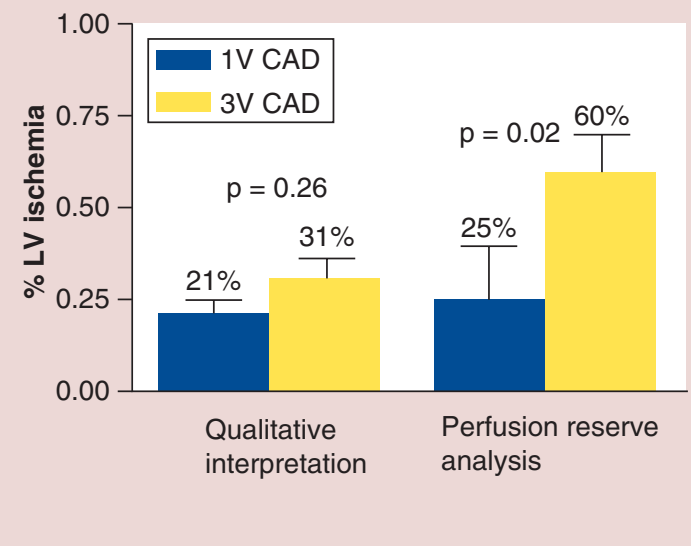

Figure 4. Quantitative perfusion analysis on stress cardiac MRI. (A) Relationship between perfusion reserve and percentage stenosis on cardiac catheterization. (B) Comparison of qualitative perfusion analysis with perfusion reserve to assess degree of ischemia in single and multivessel CAD.

CAD: Coronary artery disease; LV: Left ventricle.

Adapted with permission from [6]. 
mean follow-up was 32 months. In patients with a positive test on follow-up, the annualized event rate, CV death and MI were 4.9, 2.8 and 2.6\%, respectively. Patients with a negative stress CMR on the other hand, had $<1 \%$ annualized event rate, CV death or nonfatal MI on follow-up. This clearly indicates that stress CMR provides excellent prognostic information in regards to risk stratification of patients with known or suspected CAD.

\section{Limitations in myocardial perfusion analysis by CMR}

Despite an expansion in the utilization of CMR perfusion in clinical settings, its practice is limited due to the lack of availability of CMR imaging expertise in many centers. This is changing as more data are being published with CMR and more imaging specialists are being trained in the techniques. The additional limiting factors with CMR perfusion are patient-related such as obesity, claustrophobia, poor gating, motion artifacts, contraindications to use of vasodilator agents and obtaining appropriate heart rate response with stress testing. The latter can usually be addressed by increasing stress agent dose, for example for adenosine.

\section{Future perspective}

Multicenter randomized controlled trials have established stress CMR to be noninferior, and even superior as compared to SPECT for CAD assessment. Recent studies have demonstrated its prognostic capacity for predicting risk of future major adverse cardiovascular events in intermediate to high-risk patients. Rest perfusion in the setting of acute MI can demonstrate MVO, which is indicative of poor functional recovery of the myocardium and poor prognosis for the patient. Ongoing studies are assessing the superiority of $3 \mathrm{~T}$ CMR scanners in perfusion assessment as well as the effectiveness of MPR measurements. With additional studies establishing the predictive utility of MVO on CMR, resting perfusion analysis may become an important prognostic tool after acute MI. The future appears very promising, in regards to technological innovation in CMR, resulting in reduced image acquisition time, automated analysis and decreased artifacts.

\section{Financial \& competing interests disclosure}

$C$ Kramer receives research support from Siemens Healthcare and is a consultant for Syncarc. Y Hamirani is NIH-funded by 5T32EB003841. The authors have no other relevant affiliations or financial involvement with any organization or entity with a financial interest in or financial conflict with the subject matter or materials discussed in the manuscript apart from those disclosed.

No writing assistance was utilized in the production of this manuscript.

\section{EXECUTIVE SUMMARY}

- Despite significant improvement in the diagnostic and treatment strategies for coronary artery disease, it remains the leading cause of morbidity and mortality in the USA.

- Noninvasive assessment is often used in symptomatic patients, prior to referral for an invasive coronary angiogram (with and without fractional flow reserve measurement) to assess for coronary artery disease.

- Cardiac MRI (CMR) is increasingly used to evaluate rest and stress perfusion, to identify myocardial ischemia and infarct with high degree of accuracy.

- CMR myocardial perfusion imaging is performed via first pass perfusion of the myocardium by using chelatedgadolinium, which is an extravascular and extracellular contrast agent.

- The strength of CMR is its ability to provide information in regards to myocardial chamber volumes, overall left ventricular ejection fraction and wall motion, myocardial perfusion abnormalities and late gadolinium-enhanced images to assess for evidence of fibrosis.

- Data from three randomized controlled trials using stress CMR is now available. These studies have confirmed the noninferior (and superior in some studies) performance of stress CMR relative to single-photon emission computed tomography in a realistic clinical environment.

- Adenosine stress CMR perfusion imaging at 1.5 T is well established. However, the potential superiority of imaging at $3 \mathrm{~T}$ has recently been tested in a handful of studies and it is speculated that $3 \mathrm{~T}$ might become the preferred CMR field strength for adenosine stress CMR in clinical practice. 


\section{References}

Papers of special note have been highlighted as:

- of interest; $\bullet \bullet$ of considerable interest

1 Atkinson DJ, Burstein D, Edelman RR. Firstpass cardiac perfusion: evaluation with ultrafast MR imaging. Radiology 174(3 Pt 1), 757-762 (1990).

2 Donahue KM, Weisskoff RM, Burstein D. Water diffusion and exchange as they influence contrast enhancement. J. Magn. Reson. Imaging 7(1), 102-110 (1997).

3 Jerosch-Herold M, Hu X, Murthy NS et al. Magnetic resonance imaging of myocardial contrast enhancement with MS-325 and its relation to myocardial blood flow and the perfusion reserve. J. Magn. Reson. Imaging 18(5), 544-554 (2003).

4 Cerqueira MD, Weissman NJ, Dilsizian V et al. Standardized myocardial segmentation and nomenclature for tomographic imaging of the heart. A statement for healthcare professionals from the Cardiac Imaging Committee of the Council on Clinical Cardiology of the American Heart Association. Circulation 105(4), 539-542 (2002).

5 Gerber BL, Raman SV, Nayak K et al. Myocardial first-pass perfusion cardiovascular magnetic resonance: history, theory, and current state of the art. J. Cardiovasc. Magn. Reson. 10, 18 (2008).

6 Patel AR, Antkowiak PF, Nandalur KR et al. Assessment of advanced coronary artery disease: advantages of quantitative cardiac magnetic resonance perfusion analysis. J. Am. Coll. Cardiol. 56(7), 561-569 (2010).

7 Gebker R, Jahnke C, Manka R et al. Additional value of myocardial perfusion imaging during dobutamine stress magnetic resonance for the assessment of coronary artery disease. Circ. Cardiovasc. Imaging 1(2), 122-130 (2008).

8 Nijveldt R, Hofman MB, Hirsch A et al. Assessment of microvascular obstruction and prediction of short-term remodeling after acute myocardial infarction: cardiac MR imaging study. Radiology 250 (2), 363-370 (2009).

9 Ørn S, Manhenke C, Greve OJ et al. Microvascular obstruction is a major determinant of infarct healing and subsequent left ventricular remodelling following primary percutaneous coronary intervention. Eur. Heart J. 30(16), 1978-1985 (2009)

- $\quad$ Assessed cardiac MRI (CMR) microvascular obstruction at different time points postmyocardial infarction to determine appropriate timing for imaging that would predict major adverse cardiac events.

10 Nijveldt R, Beek AM, Hirsch A et al. Functional recovery after acute myocardial infarction: comparison between angiography, electrocardiography, and cardiovascular magnetic resonance measures of microvascular injury. J. Am. Coll. Cardiol. 52(3), 181-189 (2008).

11 Wu KC, Zerhouni EA, Judd RM et al. Prognostic significance of microvascular obstruction by magnetic resonance imaging in patients with acute myocardial infarction. Circulation 97( 8), 765 -773 (1998).

- One of the pioneer papers assessing utility of microvascular obstruction by CMR.

12 Choi CJ, Haji-Momenian S, Choudhri AF et al. Infarct involution and improved function during healing of acute myocardial infarction: the role of microvascular obstruction. J. Cardiovasc. Magn. Reson. 6(4), 917-925 (2004)

13 Lund GK, Stork A, Saeed M et al. Acute myocardial infarction: evaluation with first pass enhancement and delayed enhancement MR imaging compared with 210T1SPECT imaging. Radiology 232, 49-57 (2004).

14 Nandalur KR, Dwamena BA, Choudri AF et al. Diagnostic performance of stress cardiac magnetic resonance imaging in the detection of coronary artery disease: a meta-analysis. J. Am. Coll. Cardiol. 50, 1343-1353 (2007).

- Meta-analysis of old studies.

15 de Jong MC, Genders TS, Van Geuns RJ, Moelker A, Hunink MG. Diagnostic performance of stress myocardial perfusion imaging for coronary artery disease: a systemic review and meta-analysis. Eur. Radiol. 22(9), 1881-1895 (2012).

16 Schwitter J, Wacker CM, van Rossum AC et al. MR-IMPACT: comparison of perfusion-cardiac magnetic resonance with single-photon emission computed tomography for the detection of coronary artery disease in a multicentre, multivendor, randomized trial. Eur. Heart J. 29(4), 480-489 (2008).

17 Greenwood JP, Maredia N, Younger JF et al. Cardiovascular magnetic resonance and single-photon emission computed tomography for diagnosis of coronary heart disease (CE-MARC): a prospective trial. Lancet 379 (9814), 453-460 (2012).

-• First randomized trial comparing CMR stress perfusion with single photon emission computed tomography.

18 Schwitter J, Wacker CM, Wilke N et al. MR-IMPACT II: magnetic resonance imaging for myocardial perfusion assessment in coronary artery disease trial: perfusioncardiac magnetic resonance vs. single-photon emission computed tomography for the detection of coronary artery disease: a comparativemulticentre, multivendor trial. Eur. Heart J. 34(10), 775-781 (2013)

-• Randomized controlled trial comparing stress CMR with single-photon emission computed tomography.

19 Hussain ST, Paul M, Plein S et al. Design and rationale of the MR-INFORM study: stress perfusion cardiovascular magnetic resonance imaging to guide themanagement of patients with stable coronary artery disease. J. Cardiovasc. Magn. Reson. 14, 65 (2012).

- Randomized trial that will compare CMR stress perfusion with fractional flow reserve on cardiac catheterization.

20 Bettencourt N, Chiribiri A, Schuster A et al. Cardiac magnetic resonance myocardial perfusion imaging for detection of functionally significant obstructive coronary artery disease: a prospective study. Int. J. Cardiol. 168(2), 765-773 (2012).

21 Manka R, Jahnke C, Paetsch I et al. Dynamic 3-dimensional stress cardiac magnetic resonance perfusion imaging: detection of coronary artery disease and volumetry of myocardial hypoenhancement before and after coronary stenting. J. Am. Coll. Cardiol. 57, 437-44 (2011).

22 Walcher T, Ikuye K, Rottbauer W, Wohrie J, Bernhardt P. Is contrast-enhanced cardiac resonance imaging at $3 \mathrm{~T}$ superior to $1.5 \mathrm{~T}$ for detection of coronary artery disease? Int. J. Cardiovasc. Imaging 29, 355-361 (2013).

- Important trial comparing $3 \mathrm{~T}$ versus $1.5 \mathrm{~T}$ CMR perfusion.

23 Jogiya R, Kozerke S, Morton G et al. Validation of dynamic3-

dimensionalwholeheart magnetic resonance myocardial perfusion imaging against fractional flow reserve for the detection of significant coronary artery disease. J. Am. Coll. Cardiol. 60 (8), 756-765(2012).

24 Pack NA, DiBella EV. Comparison of myocardial perfusion estimates from dynamic contrast-enhanced magnetic resonance imaging with four quantitative analysis methods. Magn. Reson. Med. 64(1), 125-137 (2010).

25 Jerosch-Herold M, Wilke N, Stillman AE. Magnetic resonance quantification of the myocardial perfusion reserve with a Fermi function model for constrained deconvolution. Med. Phys. 25, 73-84 (1998). 
26 Morton G, Chiribiri A, Ishida M et al. Quantification of absolute myocardial perfusion in patients with coronary artery disease: comparison between cardiovascular magnetic resonance and positron emission tomography. J. Am. Coll. Cardiol. 60(16), 1546-1555 (2012).

27 Karamitsos TD, Arnold JR, Pegg TJ et al. Patients with syndrome $\mathrm{X}$ have normal transmural myocardial perfusion and oxygenation: a 3-T cardiovascular megnetic resonance imaging study. Circ. Cardiovasc. Imaging 5(2), 194-200 (2012).

28 Larghat A, Biglands J, Maredia N et al. Endocardial and epicardial myocardial perfusion determined by semi-quantitative and quantitative myocardial perfusion magnetic resonance. Int. J. Cariovasc. Imaging 28(6), 1499-1511 (2012).

29 Tarroni G, Corsi C, Antkowiak PF et al. Myocardial perfusion: near-automated evaluation from contrast-enhanced MR images obtained at rest and during vasodilator stress. Radiology 265(2), 576-583 (2012).

30 Bhave NM, Freed BH, Yodwut C et al. Considerations when measuring myocardialperfusion reserve by cardiovascular magnetic resonance using regadenoson. J. Cardiovasc. Magn. Reson. 14, 89 (2012).

31 Morton G, Jogiya R, Plein S et al. Quantitative cardiovascular magnetic resonanceperfusion imaging: inter-study reproducibility. Eur. Heart J. Cardiovasc. Imaging 13(11), 954-960 (2012).

32 Harrison A, Adluru G, Damal K et al. Rapid ungated myocardial perfusioncardiovascular magnetic resonance: preliminary diagnostic accuracy. J. Cardiovasc. Magn. Reson. 15(1), 26 (2013).

33 Ge L, Kino A, Griswold M et al. Freebreathing myocardial perfusion MRIusing SW-CG-HYPR and motion correction. Magn. Reson. Med. 64, 1148-1154 (2010).

34 Motwani M, Maredia N, Fairbairn TA et al. High-resolution versus standard resolutioncardiovascular MR myocardial perfusion imaging for the detection of coronaryartery disease. Circ. Cardiovasc. Imaging 5, 306-313 (2012).

35 Ma H, Yang J, Liu J et al. Myocardial perfusion magnetic resonanceimaging using sliding-window conjugate-gradient highly constrainedback-projection reconstruction for detection of coronary artery disease. Am. J. Cardiol. 109, 1137-1141 (2012).

36 Morton G, Ishida M, Schuster A et al. Perfusion cardiovascular magneticresonance: comparison of an advanced, high resolution and a standard sequence. J. Cardiovasc. Magn. Reson. 9(14), 34 (2012).

37 Gebker R, Jahnke C, Manka R et al. High spatial resolution myocardial perfusion imaging during high dose dobutamine/ atropine stress magneticresonance using $\mathrm{k}-\mathrm{t}$ SENSE. Int. J. Cardiol. 158(3), 411-416 (2012).

38 Lipinski MJ, McVey CM, Berger JS, Kramer CS, Salerno M. Prognostic value of stress cardiacmagnetic resonance imaging in patients with known or suspected coronary arterydisease: a systematic review and meta-analysis. J. Am. Coll. Cardiol. 62(9), 826-838 (2013). 